\title{
Short Term Outcome of Saccharomyces boulardii impact the Quality of Life in Patients with Inflammatory Bowel Diseases: A Multicenter, Double Blind, Placebo-Controlled Trial
}

\author{
Srdjan Markovic ${ }^{1,2 *}$, Nikola Panic ${ }^{2,3}$, Branimir Zogovic ${ }^{4}$, Ana Kalaba ${ }^{1}$, \\ Marijana Jankovic ${ }^{1}$, Tamara Knezevic Ivanovski ${ }^{1}$, Dino Tarabar $^{3}$ and \\ Petar Svorcan $^{1,2}$ \\ ${ }^{1}$ Department of Gastroenterology, Clinical Center Zvezdara, Belgrade, Serbia \\ ${ }^{2}$ School of Medicine, University of Belgrade, Serbia \\ ${ }^{3}$ Digestive Endoscopy Department, University Clinic "Dr Dragisa Misovic", Belgrade, \\ Serbia \\ ${ }^{4}$ Department of Surgery, Royal Prince Alfred Hospital, Camperdown, NSW Australia \\ *Corresponding Author: Srdjan Markovic, Department of Gastroenterology, \\ University Hospital Medical Center "Zvezdara”, School of Medicine, University of \\ Belgrade, Belgrade, Serbia.
}

Received: January 11, 2021

Published: February 17, 2021

(C) All rights are reserved by Srdjan

Markovic., et al.

\begin{abstract}
Introduction: Inflammatory bowel disease (IBD) and irritable bowel syndrome (IBS) have high burden on quality of life. Dietary supplements such as probiotics have been shown to have beneficial effects on the course of IBS and IBD. So far, Saccharomyces boulardii is the only probiotic yeast with a documented health promoting profile.

Aim of the Study: The main aim of the study is to investigate the effects to quality of life in patients with IBD in remission as well as patients with mild to moderate ulcerative colitis (UC) and Crohn disease (CD) when treated with Saccharomyces boulardii. The secondary aim is to investigate the safety of the probiotic preparation in patients with IBD.

Methods: This is a double-blind, placebo-controlled study. Adult subjects who satisfied the Rome IV criteria and had colonoscopy in the last six months were randomised into two groups: conventional IBD therapy with probiotic Saccharomyces boulardii (Bulardi 500) or placebo for one-month. Patients completed the WPAI-GH Questionnaire, Visual Analogue Productivity Scale, Therapy Satisfaction Questionnaire, SIBDQ before initiating probiotic and shortly after completing one-month therapy.

Results: Patients reported improvements in the quality of their personal and professional lives following the use of Saccharomyces boulardii. Saccharomyces boulardii has a safe profile. Some effects are similar to those caused by placebo.

Conclusion: Saccharomyces boulardii has many positive effects in patients with inflammatory bowel disease. Longer treatment and endoscopic assessments are needed for objective assessment of the effects of Saccharomyces boulardii.
\end{abstract}

Keywords: Inflammatory Bowel Disease; Irritable Bowel Syndrome; Saccharomyces boulardii; Bulardi 500

\section{Abbreviations}

CD: Crohn's Disease; IBD: Inflammatory Bowel Disease; IBS: Irritable Bowel Syndrome; UC: Ulcerative Colitis

\section{Introduction}

The origins and behaviour of inflammatory bowel diseases (IBD) remain unknown. However, significant progress is seen in the 
diagnosis and management of both Crohn's disease and ulcerative colitis [1,2]. Once IBD is clinically suspected, diagnosis and disease classification are made endoscopically and histologically [2,3]. Disease suppression and remission is typically done via the 'step up' or the 'top-down approach. The 'step up' approach escalates IBD medications from low to high potency based on the clinical response. The 'top down' approach introduces the most potent medications at the beginning of treatment [4]. The standard panel used in IBD management includes 5-ASA, immunomodulators, corticosteroids, anti-TNF monoclonal antibodies and biologics $[7,8]$. The ultimate goal in IBD management is to stop inflammation at mucosal level as this reduces the intestinal and extraintestinal complications of the disease. Poorly controlled IBD increases the risk of surgical intervention, colorectal cancer and extraintestinal malignancies [1]. For this reason, the 'treat to target' strategy was designed to achieve endoscopic remission using combination therapy (immunomodulators and anti-TNF agents) in patients with early disease and no complications [4-6]. Disease remission involves the absence of disease symptoms as well as intact mucosa or mild disease activity on endoscopy $[3,5,6]$. If endoscopy suggests remission and the patient has ongoing symptoms, the existence of irritable bowel syndrome (IBS) should be considered [7].

In addition to pharmaceuticals, there is an increasing interest in the use of probiotics, i.e. bacteria (Lactobacillus spp., Bifidobacteria spp.) and yeast ( $S$. boulardii) as biotherapeutics in the management of both IBD and IBS $[8,9]$. The efficacy of $S$. boulardii was first shown during the 1923 cholera epidemic [10], while recent studies suggest that it is effective in the treatment of antibiotic-related diarrhea, Clostridium difficile colitis, hepatic encephalopathy as well as IBS and IBD $[11,12]$. The value of probiotics including $S$. boulardii is in their ability to repair epithelium, reduce visceral hypersensitivity, dampen the mucosal inflammatory response and improve the gut microbiome balance $[13,14]$.

\section{Aim of the Study}

The aim of this study was to investigate the efficacy of $S$. boulardii against placebo on the quality of life of patients with both IBD and IBS-like symptoms.

\section{Materials and Methods \\ Patient selection}

Patients with confirmed histological and endoscopical diagnosis of ulcerative colitis and Crohn disease were included in the study. Symptom-free patients, patients with clinical signs of IBS or clinical/endoscopic signs of mild to moderate IBD were selected. The study was approved by the local ethics committee and all patients provided an informed consent.

The Endoscopic Mayo Subscore for ulcerative colitis and the Simple Endoscopic Score for Crohn's Disease were used to assess endoscopic activity (SES-CD). Disease activity in the Mayo subscore is graded from 0 to 3 (0-normal mucosa, 1-mild activity, 2-moderate activity, 3-extremely active disease), where values $\leq 1$ indicate remission. SES-CD (Simple Endoscopic Score-Crohn's Disease) counts the cumulative intestinal mucosal changes, ulcerated areas, disease affected areas and stenoses in affected segments. Scores of 0 - 2 indicate remission, 3 - 6 mild, 7 - 15 moderate and values > 15 indicate high disease activity.

Patients with IBS-like symptoms and IBD patients in endoscopic remission had to satisfy the Rome IV criteria in order to be included in the study. The Rome IV criteria states that patients should have recurrent abdominal pain at least once a week for minimum of three months. In addition to pain, patients need to have at least two associated symptoms: pain with defecation, change in stool appearance or form and change in frequency of stools.

Patients were asked to fill in the Short Inflammatory Bowel Disease Questionnaire (SIBDQ), the Treatment Satisfaction Questionnaire for Medication (TSQM), the Work productivity and activity impairment questionnaire (WPAI-GH) and Visual scale of productivity (VAS-P) questionnaires during the follow up period.

Study inclusion criteria were age between 18 and 80 years, signed informed consent, Crohn's disease or ulcerative colitis diagnosis for at least three months, patients with colonoscopy confirmed remission in the preceding six months that satisfied the Roma IV criteria, patients with mild to moderate disease activity based on endoscopic and clinical finding prior to study enrollment.

Study exclusion criteria were colonoscopy older than last six months, highly active disease on endoscopy, lack of pathohistological confirmation of the diagnosis of IBD, concurrent participation in another study and refusal to participate in this study.

\section{Study design}

This is a multicenter, double-blind, placebo-controlled study. The Clinical Center "Zvezdara", the Clinical Center "Dragisa Miso- 
vic" and the Clinical Center of Serbia were the three tertiary hospitals that participated in the study. The survey was conducted between April and June 2019. Subjects were randomly divided into two groups: the first received the Bulardi 500 probiotic with conventional IBD therapy and the second received placebo with conventional IBD therapy.

S. boulardii manufactured as a dietary supplement 'Bulardi 500' and placebo preparations were supplied by AbelaPharm Pty. Ltd. Belgrade.

\section{Follow up}

The follow up lasted four weeks and included a screening period and three visits. The first seven days of the study were the screening period where patients signed the consent form, their medical records were collected, and clinical disease assessment questionnaires were completed. The first visit consisted of therapy initiation (Bulardi 500 or placebo) and completion of quality of life questionnaire (patient reported outcomes). The second visit took place one week after initiation of therapy and consisted of a telephone call to evaluate for possible side effects and completion of the clinical activity assessment questionnaire. The third and final visit, which occurred four weeks after the initiation of therapy, consisted of final evaluation and completion of all questionnaires.

\section{Statistical analysis}

Descriptive analysis (proportion and mean \pm SD) was used for categorical and quantitative variables, respectively. T-test, chisquare test and signed-rank test were used to compare groups. Analyses were conducted using Stata software (StataCorp). 2019. Stata Statistical Software: Release 13. College Station, TX: StataCorp LP).

\section{Results and Discussion}

159 patients were included in the study after final selection. 55 patients had Crohn's disease, 62 patients had ulcerative colitis and 42 IBD patients fulfilled the criteria for IBS.

55 patients with mild Crohn disease: Upon randomisation, 28 were allocated to the probiotic group and 27 patients to the placebo group. Demographic and clinical characteristics are shown in table 5. There was no significant difference in the demographic or clinical characteristics between the groups at the time of inclusion (Table 5). No adverse report has been reported during study period in both groups.
Both groups showed significant improvement in relation to all individual elements of the questionnaire as well as the total score once therapy was completed (Table 6). In regard to the 'Patient Satisfaction with Therapy' questionnaire, patients in the probiotic group reported significant improvement in relation to question 1 - 3 and 12 - 14, while the patients in the placebo group reported significant improvement in relation to all questions except question 11 (Table 7). Both groups reported significant improvement in work efficiency (WPAI-GH questionnaire; placebo group $\mathrm{P}<0.001$, probiotic group $\mathrm{P}<0.05$ ) and regular activities (placebo group $\mathrm{P}$ $<0.001$, probiotic group $\mathrm{P}<0.001$ ). There was no statistical difference in VAS productivity for both groups. After analyzing the HBI score, there was statistically significant improvement in both groups regarding elements $\mathrm{A}, \mathrm{B}$ and $\mathrm{C}$ as well as the total HBI score ( $\mathrm{P}<0.001$ for all comparisons).

62 patients with mild ulcerative colitis: 33 and 29 patients were randomised to the probiotic and placebo group, respectively. There was no significant difference in any of the demographic or clinical characteristics between the groups at the time of inclusion (Table 8). No adverse effects were noted in either group. The probiotic group performed significantly better with question 10 in the quality-of-life measurement prior to start of treatment $(\mathrm{P}<0.05)$. The probiotic group also had significant improvement in all elements of the questionnaire as well as the general score (Table 9). The placebo group had significant improvement in all features except question 10 (Table 9). Both groups were satisfied with the provided therapy in relation to all features measured by the questionnaire (Table 10). Both groups of patients reported significant improvement in relation to work efficiency (WPAI-GH questionnaire; placebo group $\mathrm{P}<0.001$, probiotic group $\mathrm{P}<0.05$ ) and regular activities (placebo group $\mathrm{P}<0.001$, probiotic group $\mathrm{P}<0.001$ ). There was no statistical difference in VAS productivity for both groups. After analyzing Mayo score, statistically significant improvement in both groups was observed regarding all the elements measured (Table 11).

42 patients with IBS: 23 and 19 patients randomised to the probiotic and placebo group, respectively.

There was no difference in any of the demographic or clinical characteristics between the groups at the time of inclusion (Table 9). Patients in the probiotic group had significantly longer duration of disease at the induction $(7.5 \pm 4.6$ vs. $4.4 \pm 2.8, \mathrm{P}<0.05)$. Significant improvement in quality of life of patients in the probi- 
Short Term Outcome of Saccharomyces boulardii impact the Quality of Life in Patients with Inflammatory Bowel Diseases: A Multicenter, Double Blind, Placebo-Controlled Trial

otic group, measured by questions 1,3-6 and 8, was observed after one month of therapy (Table 2). Patients in the placebo group also reported significant improvement according to questions 1 , 3 - 8 and 10 as well as in relation to total score measured by the questionnaire (Table 2). Both Probiotic and placebo group showed significant improvement in all of the elements of patient's satisfaction with therapy during the study (Table 3). No adverse report has been reported during study period in both groups. Table 4 reports changes in answer to WPAI-GH questionnaire as a consequence of the therapy (Table 4). The work efficiency significant improvement was observed in the Probiotic group after the therapy $(p=0.033)$ (Table 4). There was no significant change in all the other elements measured both in Probiotic and placebo group (Table 4).

\begin{tabular}{|c|c|c|c|}
\hline & Probiotic & Placebo & $\mathbf{P}$ \\
\hline Age & $42.96 \pm 15.79$ & $40.32 \pm 12.45$ & 0.56 \\
\hline \multicolumn{4}{|l|}{ Gender } \\
\hline Female & $13(59.01 \%)$ & $9(40.91 \%)$ & \multirow{2}{*}{0.55} \\
\hline Male & $10(50.00 \%)$ & $10(50.00 \%)$ & \\
\hline Disease duration & $7.51 \pm 4.58$ & $4.44 \pm 2.83$ & 0.025 \\
\hline \multicolumn{4}{|l|}{ IBD subtype } \\
\hline Ulcerative colitis & $15(62.50 \%)$ & $9(37.50 \%)$ & \multirow{2}{*}{0.25} \\
\hline Crohn's disease & $8(34.78 \%)$ & $10(55.56 \%)$ & \\
\hline Employed & 13 (59.09\%) & 17 (89.47\%) & 0.029 \\
\hline
\end{tabular}

Table 1: Demographic and clinical characteristics of IBD patients in remission fulfilling criteria for IBS included in the study.

\begin{tabular}{|l|c|c|}
\hline & Probiotic & Placebo \\
\hline Question 1 & $\mathrm{P}=0.0001$ & $\mathrm{P}=0.011$ \\
\hline Question 2 & $\mathrm{P}=0.11$ & $\mathrm{P}=0.21$ \\
\hline Question 3 & $\mathrm{P}=0.029$ & $\mathrm{P}=0.029$ \\
\hline Question 4 & $\mathrm{P}=0.0046$ & $\mathrm{P}=0.0017$ \\
\hline Question 5 & $\mathrm{P}=0.033$ & $\mathrm{P}=0.0017$ \\
\hline Question 6 & $\mathrm{P}=0.000$ & $\mathrm{P}=0.011$ \\
\hline Question 7 & $\mathrm{P}=0.11$ & $\mathrm{P}=0.046$ \\
\hline Question 8 & $\mathrm{P}=0.0065$ & $\mathrm{P}=0.0021$ \\
\hline Question 9 & $\mathrm{P}=0.19$ & $\mathrm{P}=0.073$ \\
\hline Question 10 & $\mathrm{P}=0.38$ & $\mathrm{P}=0.006$ \\
\hline Total score & $\mathrm{P}=0.14$ & $\mathrm{P}=0.0056$ \\
\hline
\end{tabular}

Table 2: Effects of therapy in probiotic and placebo group on quality of life of IBD patients in remission measured by questionnaire.

\begin{tabular}{|l|c|c|}
\hline & Probiotic & Placebo \\
\hline Question 1 & $\mathrm{P}=0.0009$ & $\mathrm{P}=0.0065$ \\
\hline Question 2 & $\mathrm{P}=0.0001$ & $\mathrm{P}=0.0005$ \\
\hline Question 3 & $\mathrm{P}=0.0001$ & $\mathrm{P}=0.0005$ \\
\hline Question 9 & $\mathrm{P}=0.0021$ & $\mathrm{P}=0.0112$ \\
\hline Question 10 & $\mathrm{P}=0.010$ & $\mathrm{P}=0.0017$ \\
\hline Question 11 & $\mathrm{P}=0.0112$ & $\mathrm{p}=0.0001$ \\
\hline Question 12 & $\mathrm{P}=0.0002$ & $\mathrm{P}=0.0001$ \\
\hline Question 13 & $\mathrm{P}=0.0001$ & $\mathrm{P}=0.0039$ \\
\hline Question 14 & $\mathrm{P}=0.0065$ & $\mathrm{P}=0.0001$ \\
\hline
\end{tabular}

Table 3: Effects of treatment of IBD patients in remission $f$ ulfilling criteria for IBS on satisfaction with therapy measured by the questionnaire.

\begin{tabular}{|l|c|c|}
\hline & Probiotic & Placebo \\
\hline No of hours of sick leave sati & $\mathrm{P}=0.99$ & $/$ \\
\hline No of work hours & $\mathrm{P}=0.56$ & $\mathrm{P}=0.76$ \\
\hline Work efficiency & $\mathrm{P}=0.033$ & $\mathrm{P}=0.051$ \\
\hline Regular activities & $\mathrm{P}=0.36$ & $\mathrm{P}=0.09$ \\
\hline VAS productivity & $\mathrm{P}=0.13$ & $\mathrm{P}=0.39$ \\
\hline
\end{tabular}

Table 4: Effects of therapy on changes in WPAI-GH questionnaire in IBD patients in remission fulfilling criteria for IBS.

\begin{tabular}{|c|c|c|c|}
\hline & Probiotic & Placebo & $\mathbf{P}$ \\
\hline Age & $35.58 \pm 10.38$ & $40.74 \pm 16.50$ & 0.18 \\
\hline \multicolumn{4}{|l|}{ Gender } \\
\hline Female & $13(46.43 \%)$ & 15 (55.56\%) & \multirow{2}{*}{0.50} \\
\hline Male & $15(53.57 \%)$ & 12 (44.44\%) & \\
\hline \multicolumn{4}{|l|}{ Localization } \\
\hline Ileum/Ileocolon & $23(80.77 \%)$ & $24(92.31 \%)$ & \multirow{2}{*}{0.07} \\
\hline Colon & $5(19.23 \%)$ & $2(7.69 \%)$ & \\
\hline Perianal disease & $10(35.71 \%)$ & $5(19.23 \%)$ & 0.18 \\
\hline Surgery & $6(21.43 \%)$ & 7 (25.93\%) & 0.70 \\
\hline Disease duration & $7.14 \pm 5.48$ & $7.84 \pm 5.94$ & 0.67 \\
\hline Employed & $14(50.00 \%)$ & $14(51.85 \%)$ & 0.89 \\
\hline
\end{tabular}

Table 5: Demographic and clinical characteristics of CD patients included in the study. 
Short Term Outcome of Saccharomyces boulardii impact the Quality of Life in Patients with Inflammatory Bowel Diseases: A Multicenter, Double Blind, Placebo-Controlled Trial

\begin{tabular}{|l|c|c|}
\hline & Probiotic & Placebo \\
\hline Question 1 & $\mathrm{P}=0.0002$ & $\mathrm{P}=0.000$ \\
\hline Question 2 & $\mathrm{P}=0.000$ & $\mathrm{P}=0.000$ \\
\hline Question 3 & $\mathrm{P}=0.000$ & $\mathrm{P}=0.000$ \\
\hline Question 4 & $\mathrm{P}=0.0012$ & $\mathrm{P}=0.000$ \\
\hline Question 5 & $\mathrm{P}=0.000$ & $\mathrm{P}=0.000$ \\
\hline Question 6 & $\mathrm{P}=0.0001$ & $\mathrm{P}=0.000$ \\
\hline Question 7 & $\mathrm{P}=0.0002$ & $\mathrm{P}=0.016$ \\
\hline Question 8 & $\mathrm{P}=0.000$ & $\mathrm{P}=0.000$ \\
\hline Question 9 & $\mathrm{P}=0.000$ & $\mathrm{P}=0.000$ \\
\hline Question 10 & $\mathrm{P}=0.0003$ & $\mathrm{P}=0.000$ \\
\hline Total score & $\mathrm{P}=0.000$ & $\mathrm{P}=0.000$ \\
\hline
\end{tabular}

Table 6: Effects of therapy in probiotic and placebo group on quality of life of CD patients measured by questionnaire.

\begin{tabular}{|l|c|c|}
\hline & Probiotic & Placebo \\
\hline Question 1 & $\mathrm{P}=0.000$ & $\mathrm{P}=0.000$ \\
\hline Question 2 & $\mathrm{P}=0.000$ & $\mathrm{P}=0.000$ \\
\hline Question 3 & $\mathrm{P}=0.000$ & $\mathrm{P}=0.000$ \\
\hline Question 9 & $\mathrm{P}=0.23$ & $\mathrm{P}=0.031$ \\
\hline Question 10 & $\mathrm{P}=0.34$ & $\mathrm{P}=0.016$ \\
\hline Question 11 & $\mathrm{P}=0.25$ & $\mathrm{P}=0.11$ \\
\hline Question 12 & $\mathrm{P}=0.000$ & $\mathrm{P}=0.000$ \\
\hline Question 13 & $\mathrm{P}=0.000$ & $\mathrm{P}=0.000$ \\
\hline Question 14 & $\mathrm{P}=0.000$ & $\mathrm{P}=0.000$ \\
\hline
\end{tabular}

Table 7: Effects of treatment of CD patients on satisfaction with therapy measured by the questionnaire.

\begin{tabular}{|c|c|c|c|}
\hline & Probiotic & Placebo & $\mathbf{P}$ \\
\hline Age & $48.84 \pm 16.00$ & $47.93 \pm 16.12$ & 0.83 \\
\hline \multicolumn{4}{|l|}{ Gender } \\
\hline Female & $16(48.48 \%)$ & $20(31.03 \%)$ & \multirow{2}{*}{0.10} \\
\hline Male & $17(51.52 \%)$ & $9(68.97 \%)$ & \\
\hline Disease duration & $6.96 \pm 5.68$ & $7.16 \pm 5.56$ & 0.87 \\
\hline \multicolumn{4}{|l|}{ UC localization } \\
\hline Distal & $11(34.38 \%)$ & $12(42.86 \%)$ & \multirow{2}{*}{0.50} \\
\hline Extensive & $21(65.63 \%)$ & $16(57.14 \%)$ & \\
\hline \multicolumn{4}{|l|}{ Smoking } \\
\hline Never smoker & $24(72.73 \%)$ & $23(79.31 \%)$ & \multirow{3}{*}{0.49} \\
\hline Former smoker & $8(24.24 \%)$ & $4(13.79 \%)$ & \\
\hline Active smoker & $1(3.03 \%)$ & $2(6.90 \%)$ & \\
\hline Family history of UC & $31(96.88 \%)$ & $27(100.00 \%)$ & 0.35 \\
\hline Employed & $16(48.48 \%)$ & $14(48.24 \%)$ & 0.97 \\
\hline
\end{tabular}

Table 8: Demographic and clinical characteristics of UC patients.

\begin{tabular}{|l|l|l|}
\hline & Probiotic & Placebo \\
\hline Question 1 & $\mathrm{P}=0.000$ & $\mathrm{P}=0.000$ \\
\hline Question 2 & $\mathrm{P}=0.000$ & $\mathrm{P}=0.000$ \\
\hline Question 3 & $\mathrm{P}=0.000$ & $\mathrm{P}=0.000$ \\
\hline Question 4 & $\mathrm{P}=0.000$ & $\mathrm{P}=0.000$ \\
\hline Question 5 & $\mathrm{P}=0.000$ & $\mathrm{P}=0.000$ \\
\hline Question 6 & $\mathrm{P}=0.000$ & $\mathrm{P}=0.000$ \\
\hline Question 7 & $\mathrm{P}=0.031$ & $\mathrm{P}=0.062$ \\
\hline Question 8 & $\mathrm{P}=0.000$ & $\mathrm{P}=0.000$ \\
\hline Question 9 & $\mathrm{P}=0.000$ & $\mathrm{P}=0.000$ \\
\hline Question 10 & $\mathrm{P}=0.001$ & $\mathrm{P}=0.000$ \\
\hline Total score & $\mathrm{P}=0.000$ & $\mathrm{P}=0.000$ \\
\hline
\end{tabular}

Table 9: UC patients and their quality of life as measured by the questionnaire.

\begin{tabular}{|l|c|c|}
\hline & Probiotic & Placebo \\
\hline Question 1 & $\mathrm{P}=0.000$ & $\mathrm{P}=0.000$ \\
\hline Question 2 & $\mathrm{P}=0.000$ & $\mathrm{P}=0.000$ \\
\hline Question 3 & $\mathrm{P}=0.000$ & $\mathrm{P}=0.000$ \\
\hline Question 9 & $\mathrm{P}=0.0001$ & $\mathrm{P}=0.0001$ \\
\hline Question 10 & $\mathrm{P}=0.000$ & $\mathrm{P}=0.0001$ \\
\hline Question 11 & $\mathrm{P}=0.0001$ & $\mathrm{P}=0.0001$ \\
\hline Question 12 & $\mathrm{P}=0.000$ & $\mathrm{P}=0.000$ \\
\hline Question 13 & $\mathrm{P}=0.000$ & $\mathrm{P}=0.000$ \\
\hline Question 14 & $\mathrm{P}=0.000$ & $\mathrm{P}=0.000$ \\
\hline
\end{tabular}

Table 10: UC patients therapy satisfaction as measured by the questionnaire.

\begin{tabular}{|l|c|c|}
\hline & Probiotic & Placebo \\
\hline Mayo 1 & $\mathrm{P}=0.000$ & $\mathrm{P}=0.000$ \\
\hline Mayo 2 & $\mathrm{P}=0.001$ & $\mathrm{P}=0.009$ \\
\hline Mayo 3 & $\mathrm{P}=0.000$ & $\mathrm{P}=0.000$ \\
\hline Mayo Total & $\mathrm{P}=0.000$ & $\mathrm{P}=0.000$ \\
\hline
\end{tabular}

Table 11: Mayo subscore for UC patients.

Almost half of IBD patients have IBS-like symptoms and there is increasing evidence of co-existence of IBS and IBD $[15,16]$. Current theories posit that IBS-like symptoms may be caused by subclinical 
inflammation or true IBS $[16,17]$. Sometimes it may be difficult to differentiate between the two as persistent subclinical inflammation may lead to an increase in intercellular permeability and thus start off IBS-like symptoms [18]. The gut microbiota, along with inflammation, may be involved in the pathophysiology of both conditions [19]. The gut microbiota has a dynamic cycle and decreased diversity is linked to a number of conditions [20]. In the case of IBD and IBS, the gut microbiota is disturbed but it remains unclear whether this is a cause or an effect [21]. Treatment options for IBS-like symptoms in patients with IBD are the same as in patients with true IBS. These symptoms impact the quality of life, which is why establishing adequate therapy is very important [22,23]. The management of true IBS patients without IBD includes diet modification (decreased intake of fermentable oligosaccharides, disaccharides, monosaccharides and polyols, fiber supplementation or restriction), antispasmodics, prebiotics and probiotics [24,25]. Probiotics are living microorganisms that can be used to prevent and treat a wide range of gastrointestinal diseases and are particularly good at reducing bloating and pain [26,27]. Probiotics prevent colonization of pathogenic bacteria by modulating the signaling pathways of the immune system at mucosal level and by facilitating the production of antibiotic substances [28,29]. This anti-inflammatory effect is one of the main reasons why probiotics may be useful in IBD maintenance therapy [10,30]. Bacteria (Lactobacillus spp., Bifidobacteria spp.) are most commonly used as probiotics, however $S$. boulardii is a non-pathogenic yeast has found its role as a probiotic [8].

S. boulardii was the first yeast shown to be suitable for probiotic use in humans [9,31]. It prevents gut dysbiosis by suppressing the mucosal inflammation and augmenting the immune responses in digestive tract $[28,32,33]$. The anti-inflammatory spectrum of $S$. boulardii is broad. Studies have demonstrated that $S$. boulardii affects the migration of mesenteric lymph nodes T-cells, inhibits the production of proinflammatory cytokines and activates the expression of peroxisome proliferator-activated receptor-gamma (PPARgamma) that protect from gut inflammation and IBD. In addition, $S$. boulardii exerts its anti-microbial activity by suppressing the overgrowth of bacteria and host cell adherence; in the case of $C$. difficile, $S$. boulardii stimulates antibody production against toxin $A$ and augments the release of a protease that cleaves toxin $A$ and its intestinal receptor [14]. Due to the aforementioned features, some studies have also examined the possibility of using $S$. boulardii in inflammatory bowel diseases [34].
Our study found that $S$. boulardii has a number of beneficial effects on the quality of life in patients in IBD and IBS-like symptoms. Patients were satisfied with the therapy outcomes that affected their personal and professional lives. Many of these observed benefits, were, however on par or outperformed by placebo. Given the chronicity of IBD and IBS, real-life effects would be better ascertained if patients treated with $S$. boulardii are followed up over a longer period of time, ideally at least 12 months. During this period of time, endoscopic assessment can ascertain the intraluminar architectural changes following prolonged exposure to $S$. boulardii.

\section{Conclusion}

Our study found that $S$. boulardii has a number of beneficial effects on the quality of life in patients in IBD and IBS-like symptoms. Patients were satisfied with the therapy outcomes that affected their personal and professional lives. Many of these observed benefits, were, however on par or outperformed by placebo. Given the chronicity of IBD and IBS, real-life effects would be better ascertained if patients treated with $S$. boulardii are followed up over a longer period of time, ideally at least 12 months. During this period of time, endoscopic assessment can ascertain the intraluminar and factual architectural changes following prolonged exposure to $S$. boulardii.

\section{Acknowledgements}

The authors acknowledge AbelaPharm Pty. Ltd. Belgrade for supplying the study with the dietary supplement 'Bulardi 500'.

\section{Conflict of Interest}

The authors declare no conflicts of interest.

\section{Bibliography}

1. Burisch J and P Munkholm. "The Epidemiology of Inflammatory Bowel Disease". Scandinavian Journal of Gastroenterology 50.8 (2015): 942-951.

2. Van Assche G., et al. "The Second European Evidence-Based Consensus on the Diagnosis and Management of Crohn's Disease: Definitions and Diagnosis". Journal of Crohn's and Colitis 4.1 (2010): 7-27.

3. Dignass A., et al. "Second European Evidence-Based Consensus on the Diagnosis and Management of Ulcerative Colitis Part 1: Definitions and Diagnosis". Journal of Crohn's and Colitis 6.10 (2012): 965-990. 
4. Panaccione R., et al. "Review Article: Treatment Algorithms to Maximize Remission and Minimize Corticosteroid Dependence in Patients with Inflammatory Bowel Disease". Alimentary Pharmacology and Therapeutics 28.6 (2008): 674-688.

5. Ferrante M., et al. "Validation of Endoscopic Activity Scores in Patients with Crohn's Disease Based on a Post Hoc Analysis of Data from Sonic". Gastroenterology 145.5 (2013): 978-865.

6. Samuel S., et al. "Validation of the Ulcerative Colitis Colonoscopic Index of Severity and Its Correlation with Disease Activity Measures". Clinical Gastroenterology and Hepatology 11.1 (2013): 49-54.

7. Barbara G., et al. "Inflammatory Bowel Disease and Irritable Bowel Syndrome: Similarities and Differences". Current Opinion in Gastroenterology 30.4 (2014): 352-358.

8. Guslandi M., et al. "Saccharomyces boulardii in Maintenance Treatment of Crohn's Disease". Digestive Diseases and Sciences 45.7 (2000): 1462-1464.

9. Plaza-Diaz J., et al. "Evidence of the Anti-Inflammatory Effects of Probiotics and Synbiotics in Intestinal Chronic Diseases". Nutrients 9.6 (2017).

10. Rolfe VE., et al. "Probiotics for Maintenance of Remission in Crohn's Disease". The Cochrane Database of Systematic Reviews 4 (2006): Cd004826.

11. Mario Guslandi., et al. "A Pilot Trial of Saccharomyces boulardii in Ulcerative Colitis". European Journal of Gastroenterology and Hepatology 15.6 (2003): 697-698.

12. Rowland I., et al. "Current Level of Consensus on Probiotic Science--Report of an Expert Meeting--London, 23 November 2009". Gut Microbes 1.6 (2010): 436-439.

13. Rodriguez-Nogales A., et al. "Intestinal Anti-Inflammatory Effect of the Probiotic Saccharomyces boulardii in Dss-Induced Colitis in Mice: Impact on Micrornas Expression and Gut Microbiota Composition". Journal of Nutritional Biochemistry 61 (2018): 129-139.

14. Dalmasso G., et al. "Saccharomyces boulardii Inhibits Inflammatory Bowel Disease by Trapping $\mathrm{T}$ Cells in Mesenteric Lymph Nodes". Gastroenterology 131.6 (2006): 1812-1825.

15. Spiller R and G Major. "Ibs and Ibd - Separate Entities or on a Spectrum?" Nature Reviews Gastroenterology and Hepatology 13.10 (2016): 613-621.
16. Stanisic V and EM Quigley. "The Overlap between Ibs and Ibd: What Is It and What Does It Mean?" Expert Review of Gastroenterology and Hepatology 8.2 (2014): 139-145.

17. Berrill JW., et al. "Symptoms of Irritable Bowel Syndrome in Patients with Inflammatory Bowel Disease: Examining the Role of Sub-Clinical Inflammation and the Impact on Clinical Assessment of Disease Activity". Alimentary Pharmacology and Therapeutics 38.1 (2013): 44-51.

18. Keohane J., et al. "Irritable Bowel Syndrome-Type Symptoms in Patients with Inflammatory Bowel Disease: A Real Association or Reflection of Occult Inflammation?" The American Journal of Gastroenterology 105.8 (2010): 89-94.

19. Shanahan F and EM Quigley. "Manipulation of the Microbiota for Treatment of Ibs and Ibd-Challenges and Controversies". Gastroenterology 146.6 (2014): 1554-1563.

20. Gareau MG., et al. "Probiotics and the Gut Microbiota in Intestinal Health and Disease". Nature Reviews Gastroenterology and Hepatology 7.9 (2010): 503-514.

21. Nagalingam NA and SV Lynch. "Role of the Microbiota in Inflammatory Bowel Diseases". Inflammatory Bowel Diseases 18.5 (2012): 968-984.

22. Jelsness-Jorgensen LP., et al. "Coexisting Irritable Bowel-Like Symptoms in Inflammatory Bowel Disease in Remission Is Associated with Impaired Social Functioning and Increased Bodily Pain". Gastroenterology Nursing 37.4 (2014): 280-287.

23. Simren M., et al. "Quality of Life in Inflammatory Bowel Disease in Remission: The Impact of Ibs-Like Symptoms and Associated Psychological Factors". The American Journal of Gastroenterology 97.2 (2002): 389-396.

24. Bijkerk CJ., et al. "Systematic Review: The Role of Different Types of Fibre in the Treatment of Irritable Bowel Syndrome". Alimentary Pharmacology and Therapeutics 19.3 (2004): 245251.

25. Rajilic-Stojanovic M., et al. "Intestinal Microbiota and Diet in Ibs: Causes, Consequences, or Epiphenomena?" The American Journal of Gastroenterology 110.2 (2015): 278-287.

26. Curro D., et al. "Probiotics, Fibre and Herbal Medicinal Products for Functional and Inflammatory Bowel Disorders". British Journal of Pharmacology 174.11 (2017): 1426-1449. 
27. Rondanelli M., et al. "Using Probiotics in Clinical Practice: Where Are We Now? A Review of Existing Meta-Analyses". Gut Microbes 8.6 (2017): 521-543.

28. Brun P., et al. "Saccharomyces boulardii Cncm I-745 Supplementation Reduces Gastrointestinal Dysfunction in an Animal Model of Ibs". PLoS One 12.7 (2017): e0181863.

29. Girardin M and EG Seidman. "Indications for the Use of Probiotics in Gastrointestinal Diseases". Digital Distribution 29.6 (2011): 574-587.

30. Naidoo K., et al. "Probiotics for Maintenance of Remission in Ulcerative Colitis". The Cochrane Database of Systematic Reviews 12 (2011): Cd007443.

31. Ritchie ML and TN Romanuk. "A Meta-Analysis of Probiotic Efficacy for Gastrointestinal Diseases". PLoS One 7.4 (2012): e34938.

32. Palma ML., et al. "Probiotic Saccharomyces Cerevisiae Strains as Biotherapeutic Tools: Is There Room for Improvement?" Applied Microbiology and Biotechnology 99.16 (2015): 65636570.

33. Choi CH., et al. "A Randomized, Double-Blind, Placebo-Controlled Multicenter Trial of Saccharomyces boulardii in Irritable Bowel Syndrome: Effect on Quality of Life". Journal of Clinical Gastroenterology 45.8 (2011): 679-683.

34. Sivananthan K and AM Petersen. "Review of Saccharomyces boulardii as a Treatment Option in Ibd". Immunopharmacology and Immunotoxicology 40.6 (2018): 465-475.

\section{Assets from publication with us}

- Prompt Acknowledgement after receiving the article

- Thorough Double blinded peer review

- Rapid Publication

- Issue of Publication Certificate

- High visibility of your Published work

Website: www.actascientific.com/

Submit Article: www.actascientific.com/submission.php

Email us: editor@actascientific.com

Contact us: +919182824667

Citation: Srdjan Markovic., et al. "Short Term Outcome of Saccharomyces boulardii impact the Quality of Life in Patients with Inflammatory Bowel Diseases: A Multicenter, Double Blind, Placebo-Controlled Trial". Acta Scientific Gastrointestinal Disorders 4.3 (2021): 27-34. 